Bruna Antunes de Aguiar Ximenes Pereira ${ }^{1}$

Patricia Franco Cintra Schram ${ }^{2}$

Renata Cruz Soares de Azevedo ${ }^{1}$

Departamento de Psicologia Médica e Psiquiatria, Faculdade de Ciências Médicas, Universidade Estadual de Campinas. Cidade Universitária Zeferino Vaz Cidade Universitária. 13081970 Campinas SP Brasil. bruna.aax.p@gmail.com ${ }^{2}$ Division of Developmental Medicine, Boston Children's Hospital, Escola de Medicina de Harvard.

\title{
Avaliação da versão brasileira da escala CRAFFT/CESARE para uso de drogas por adolescentes
}

\author{
Evaluation of the Brazilian version of the CRAFFT/CESARE scale \\ for screening drug use by adolescentes
}

Abstract The scope of this article is to present the Brazilian version of the CRAFFT scale for screening drug use among adolescents and to assess its comprehensibility. It is a descriptive and quantitative study, which evaluated the applicability of the Brazilian version of the CRAFFT scale through a convenience sample of adolescents aged 14-21 enrolled at two technical schools. Three main aspects were evaluated: difficulty in understanding the scale; the validity of the translated scale comparing a subsample $(28,8 \%)$ that also filled out the CEBRID questionnaire; and the percentage of adolescents who scored positive on the CRAFFT/CESARE using the pre-established cutoff point. The kappa coefficient was applied to establish correspondence between questionnaires and the ROC curve was used to evaluate the psychometric differences within different cutoffs. The CRAFFT/CESARE scale was applied in $2005 \mathrm{stu}$ dents, of which 1882 are within the defined age bracket. Only $2.2 \%$ of adolescents had difficulty in understanding the questions. The specificity of the CRAFFT/CESARE scale was $73.3 \%$, and sensitivity was $87.1 \%$. The concordance degree (Kappa) was considered good (0.461). The CRAFFT/CESARE scored positive in $36.2 \%$ of the adolescents. Key words Adolescence, Primary prevention, Screening, Alcohol, Marijuana
Resumo $O$ artigo tem por objetivos apresentar a versão brasileira da escala CRAFFT de triagem de uso de drogas entre adolescentes e analisar sua compreensibilidade. Estudo descritivo e quantitativo, que avaliou versão brasileira da escala CRAFFT, e aferiu sua aplicabilidade em amostra por conveniência composta por escolares de 14 a 21 anos, regularmente matriculados em duas escolas técnicas. Foram analisados: a dificuldade de compreensão; a capacidade de avaliação através da comparação com o questionário do CEBRID aplicado em $28,8 \%$ dos estudantes; e o percentual de adolescentes considerados CRAFFT/CESARE positivos pelo ponto de corte. O coeficiente Kappa foi aplicado como medida de concordância entre os questionários e diferenças psicométricas com pontos de corte diversos foram avaliadas pela curva ROC. A escala CRAFFT/CESARE foi aplicada em 2005 alunos, sendo 1882 da faixa etária determinada. Apenas 2,2\% dos adolescentes referiu dificuldade com o entendimento das questões. A especificidade da escala CRAFFT/CESARE foi de $73,3 \%$ e a sensibilidade $87,1 \%$. O grau de concordância foi considerado bom $(0,461)$. O CRAFFT/ CESARE foi positivo em 36,2\% dos adolescentes. Palavras-chave Adolescente, Prevenção primária, Triagem, Álcool, Maconha 


\section{Introdução}

A adolescência é um período marcado por transformações biológicas, emocionais e sociais ${ }^{1}$, tais características podem contribuir para uma maior ou menor vulnerabilidade ao uso de substâncias psicoativas $(\mathrm{SPA})^{2}$. Esse uso geralmente tem início nessa fase do desenvolvimento, tornando esta etapa relevante para estratégias de prevenção e detecção precoce de uso de risco ${ }^{2,3}$.

Levantamento nacional recente apontou que o álcool e o tabaco são as substâncias mais consumidas pelos adolescentes e aproximadamente $5 \%$ iniciou a experimentação antes de 10 anos $^{4}$. Outro estudo brasileiro com adolescentes aponta que $4 \%$ já usaram maconha e $3 \%$ cocaína ao menos uma vez na vida ${ }^{5}$.

O consumo de SPA por adolescentes está associado a acidentes de trânsito ${ }^{6}$, problemas judiciais, comportamento sexual de risco ${ }^{7}$, gestação não planejada ${ }^{8}$, ferimentos não intencionais, homicídio e suicídio ${ }^{8,9}$. Embora para a maioria dos jovens, o uso de SPA não produza danos relevantes, para uma parte considerável o consumo poderá ter como consequência a evolução para o uso problemático interferindo no processo normal da adolescência ${ }^{10}$.

Associações internacionais recomendam que todos os adolescentes sejam avaliados para o uso de álcool e outras drogas como parte de uma rotina da assistência de saúde ${ }^{11}$. No entanto, existe uma baixa triagem dos adolescentes em relação ao uso de SPA, principalmente por falta de capacitação adequada para o rastreamento ${ }^{12}$.

Embora não normatize o modo de identificação dos adolescentes em uso de SPA, o Ministério da Saúde do Brasil sugere que os profissionais devem estar aptos a identificar aqueles com problemas associados ao uso de SPA, verificar sua limitação de manejo e encaminhar adequadamente ao serviço de referência ${ }^{6,13}$.

Sendo assim, métodos de triagem de fácil manejo poderiam ser incorporados nas rotinas de atendimentos ${ }^{12}$. A aplicação de instrumento validado para a triagem do uso de SPA possibilitaria a identificação precoce e a escolha de uma conduta pertinente à gravidade da situação, desde uma orientação para o adolescente até o encaminhamento para serviço habilitado a lidar com o caso $^{11}$.

O instrumento CRAFFT (acrônimo de Car; Relax; Alone; Forget; Family/Friends; Trouble) tem sido internacionalmente recomendado para rastreamento de uso de SPA nessa população ${ }^{12}$. Este instrumento já foi traduzido para diversos países $^{14-19}$ e tem sido considerado de fácil aplicação e boa capacidade psicométrica.

Em recente revisão sistemática da Cochra$n \mathrm{e}^{20}$ sobre a validação e a confiabilidade da escala CRAFFT foram encontrados 17 estudos, realizados em adolescentes na população geral ${ }^{21}$, em alistamento militar ${ }^{16}$, ambiente hospitalar ${ }^{12}$, na atenção primária à saúde ${ }^{11,19}$, serviços de emergência ${ }^{20}$ e clínicas de doenças sexualmente transmissíveis ${ }^{20}$. O CRAFFT foi considerado um bom instrumento de triagem de uso, abuso e dependência de $\mathrm{SPA}^{20}$.

Em estudos recentes ${ }^{22,23}$, o CRAFFT foi apontado como o instrumento de triagem com melhor desempenho para identificar uso de SPA entre gestantes jovens e adolescentes vítimas de trauma.

Estudo de revisão apontou que o CRAFFT apresenta os dados mais consistentes de embasamento para sua utilização na atenção primária a saúde ${ }^{24}$. A sensibilidade é muito superior ao CAGE, o qual não é recomendado para uso em adolescentes, e é similar a escalas mais extensas tais como AUDIT e POSIT ${ }^{25,26}$. Apesar de ter sido comparado com as escalas citadas ${ }^{25,26}$, o CRAFFT ainda não foi comparado a outros instrumentos já validados no Brasil, tais como o DUSI ${ }^{27}$.

Estudos apontaram que entre os diversos instrumentos utilizados para triagem do uso de SPA lícitas e ilícitas entre adolescentes ${ }^{28-30}$ o CRAFFT destaca-se como uma boa alternativa para esse fim devido a sua brevidade, boa sensibilidade, ausência de custo e da necessidade de treinamento prévio, assim como pelas diversas possibilidades de aplicação (computadorizado, aplicação por profissional da saúde e autoaplicação) ${ }^{20}$.

Os objetivos deste estudo são: avaliar uma versão brasileira da escala CRAFFT/CESARE para o Brasil, analisar sua compreensibilidade e as diferenças psicométricas com os diversos pontos de corte através da aplicação em uma amostra ampla de adolescentes estudantes. Embora este instrumento tenha sido utilizado em outros países da América do Sul ${ }^{15,26}$, trata-se do primeiro estudo nacional utilizando-o ${ }^{31}$. Existe apenas a descrição do instrumento CRAFFT em um livro nacional ${ }^{32}$.

\section{Método}

Estudo descritivo, quantitativo e transversal, que avaliou uma versão em português proposta pelos autores $^{31}$ da escala CRAFFT, aqui denominada como CRAFT/CESARE, e aferiu a sua aplicabil- 
idade em adolescentes. A população foi definida através de amostra de conveniência. Os critérios de inclusão foram: ter idade entre 14 e 21 anos, ambos os sexos, regularmente matriculados no Ensino Médio de duas escolas públicas técnicas do interior do Estado de São Paulo, no ano de 2011, possuir compreensão da língua portuguesa, estar presente na sala de aula no momento da aplicação, ter o consentimento dos pais através da assinatura do TCLE e ter lido, concordado e assinado o TCLE. O critério de exclusão foi a ausência na sala de aula no momento da aplicação.

Os instrumentos utilizados foram o questionário $\mathrm{CRAFFT}^{12} / \mathrm{CESARE}^{32}$ e o questionário do VI Levantamento Nacional sobre o consumo de drogas psicotrópicas entre estudantes do ensino fundamental e médio das redes pública e privada de ensino nas 27 capitais brasileiras-2010 .

O questionário CRAFFT é composto de 3 perguntas de filtro sobre uso de bebidas alcoólicas, maconha ou outra droga (Parte A) e seis perguntas adicionais acerca de contexto e consequências relacionadas ao uso de SPA (Parte B). Cada resposta afirmativa na Parte $\mathrm{B}$ equivale a um ponto e o ponto de corte recomendado é a pontuação igual ou superior a $2^{12}$.

O número de respostas positivas estima não apenas a presença do uso como também a magnitude do risco de problemas associados a esse uso. Trata-se de um instrumento de avalição do risco do uso de SPA e dos problemas associados, apesar de não ser uma ferramenta diagnóstica, pode nortear os profissionais de saúde para o manejo clínico, já que anexado ao instrumento existe um gráfico para auxílio do profissional que relaciona o número de respostas positivas à probabilidade do uso atual ser considerado abuso ou dependência ${ }^{12}$. A consistência interna da escala, aferida através do alpha de Cronbach, variou de 0.65 a 0,78 a depender da população estudada ${ }^{20}$.

A versão brasileira foi obtida através de um consenso entre o autor do instrumento, que enviou uma escala já traduzida para o português, e os autores deste artigo que adaptaram as perguntas para o contexto brasileiro e utilizaram a versão final para execução da pesquisa ${ }^{31}$.

O questionário do VI Levantamento Nacional sobre o consumo de drogas psicotrópicas entre estudantes do ensino fundamental e médio das redes pública e privada de ensino nas 27 capitais brasileiras- $2010^{4}$ é um questionário fechado, de autopreenchimento e sem identificação pessoal do aluno, adaptado de instrumento proposto pela OMS (Organização Mundial da Saúde). No Brasil, foi adaptado por Carlini-Cotrim et al..$^{33} \mathrm{e}$ já utilizado em seis levantamentos nacionais feitos pelo CEBRID. A escolha como instrumento comparativo foi pautada no fato de ser um instrumento validado nacionalmente, que também analisa o uso nos últimos 12 meses e por ser de autoaplicação, assim como o CRAFFT/CESARE. Este instrumento foi aplicado em uma subamostra $(28 \%)$ escolhida aleatoriamente entre os estudantes participantes, logo em seguida à aplicação do questionário CRAFFT/CESARE.

As etapas para o acesso a escala e adaptação cultural foram divididos em três fases:

Fase 1:

Contato com a equipe do autor da escala, que nos enviou uma versão em português. Após o recebimento da versão inicial, houve discussão do conteúdo com profissionais experientes no atendimento de adolescentes usuários de SPA e a partir disto foram solicitadas as seguintes adaptações com a intenção de facilitar o entendimento e a autoaplicação: troca do termo "marijuana" por maconha; inclusão do termo "chapado"; autoaplicação para avaliação da compreensão e inclusão de perguntas de fechamento para esta finalidade.

Os autores enviaram uma versão para autoaplicação do CRAFFT/CESARE. A versão final da escala contemplou as sugestões e alterações propostas.

Fase 2:

Contato com as escolas apresentando o projeto, informando os objetivos da pesquisa e solicitando a aplicação em seus alunos.

A autoaplicação das escalas foi realizada individualmente em outubro de $2011 \mathrm{em}$ ambos os colégios no período matutino, vespertino e noturno, em sala de aula após explicação dos objetivos do trabalho e preenchimento do TCLE.

Fase 3:

Foram selecionadas as questões do CEBRID que mais se aproximavam daquelas do questionário CRAFFT/CESARE. No entanto, para a pergunta B3 (Sua família ou AMIGOS já lhe disseram que você devia parar de beber ou usar menos droga?) não houve qualquer possibilidade de aproximação com alguma questão do questionário CEBRID.

O CEBRID foi considerado positivo caso houvesse uma resposta afirmativa na primeira parte e duas ou mais respostas afirmativas selecionadas para esse fim na segunda parte.

Os dados obtidos a partir dos questionários foram inseridos no programa SPSS 11.5 para Windows. A análise dos dados forneceu uma descrição sobre a compreensibilidade da versão do 
instrumento nesta população e a sua correlação com o instrumento comparativo. Foram avaliadas as taxas de respostas positivas que indicam a probabilidade de uso de risco de SPA. Para avaliar a relação entre as variáveis categóricas foi utilizado o teste Qui-quadrado, e o coeficiente Kappa foi aplicado como medida de concordância entre os questionários. Para avaliar as diferenças psicométricas com diferentes pontos de corte foi utilizada a curva ROC.

O projeto de pesquisa foi aprovado pelo Comitê de Ética em Pesquisa da FCM-UNICAMP.

\section{Resultados}

A versão final da escala CRAFFT/CESARE utilizada encontra-se na Figura 1.

A escala CRAFFT/CESARE foi aplicada em 2005 alunos. Destes, 1882 (93,9\%) estavam dentro da faixa etária de inclusão (14 a 21 anos). A idade média foi 16,7 anos; $57,2 \%$ dos alunos eram do sexo masculino; $47 \%$ estudavam no período matutino e $41 \%$ no período noturno.

Alguns alunos $(2,7 \%, \mathrm{n}=54)$ se recusarem a participar da pesquisa. A maioria destes alunos (n = 34) não justificou a razão da recusa; 20 alunos informaram porque não quiseram participar (Quadro 1).

Apenas 2,2\% dos estudantes informaram ter tido alguma dificuldade na compreensão do questionário. Uma pequena parcela de entrevistados (35 alunos) fizeram comentários e sugestões.

A Tabela 1 aponta os comentários e sugestões feitas pelos alunos.

Utilizando o ponto de corte estabelecido para a escala (ao menos uma resposta positiva na Parte A e 2 ou mais respostas positivas na Parte B) o CRAFFT/CESARE foi positivo em 682 alunos, ou seja, 36,2\% dos alunos apresentaram risco de ter problema relacionado ao uso de SPA.

$\mathrm{O}$ instrumento comparativo (questionário do CEBRID) foi aplicado em 543 alunos (28,8\%) da mesma faixa etária.

$\mathrm{O}$ instrumento comparativo foi positivo em 21,4\% dos 543 alunos.

A especificidade da escala CRAFFT/CESARE foi de $73,3 \%$ e a sensibilidade $87,1 \%$ (Tabela 2 ). A análise de concordância da escala CRAFFT/ CESARE com as perguntas escolhidas do Questionário do CEBRID apontou bom grau de concordância (Kappa 0,461).

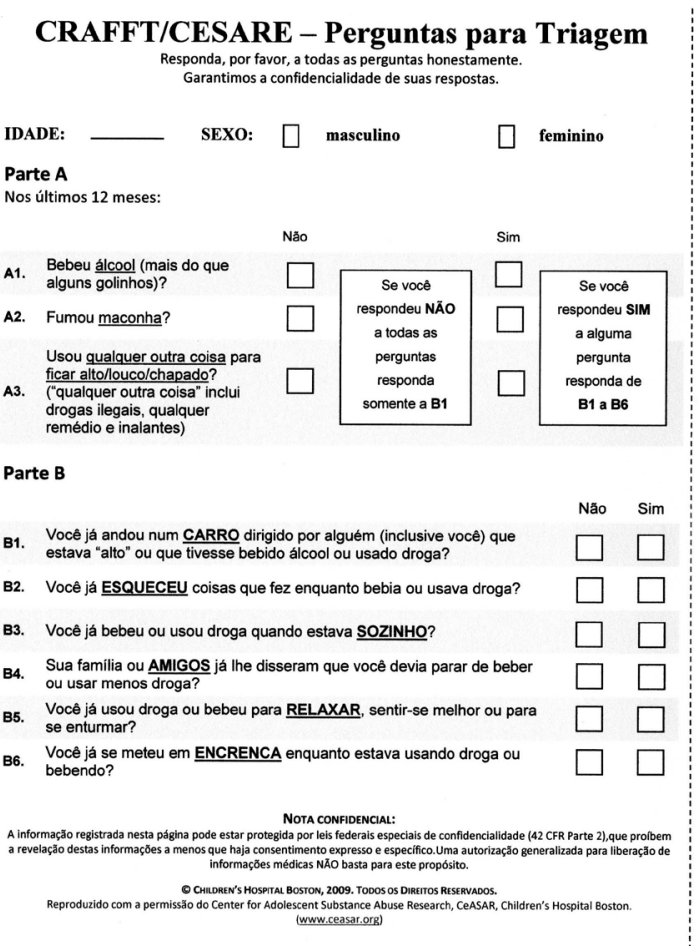

Você teve alguma dificuldade no entendimento das questões? $\square$ NÃO

Você gostaria de fazer alguma crítica ou sugestão?

Figura 1. Versão brasileira do questionário CRAFFT/CESARE. 
Quadro 1. Justificativas de recusa de participação pelos alunos.

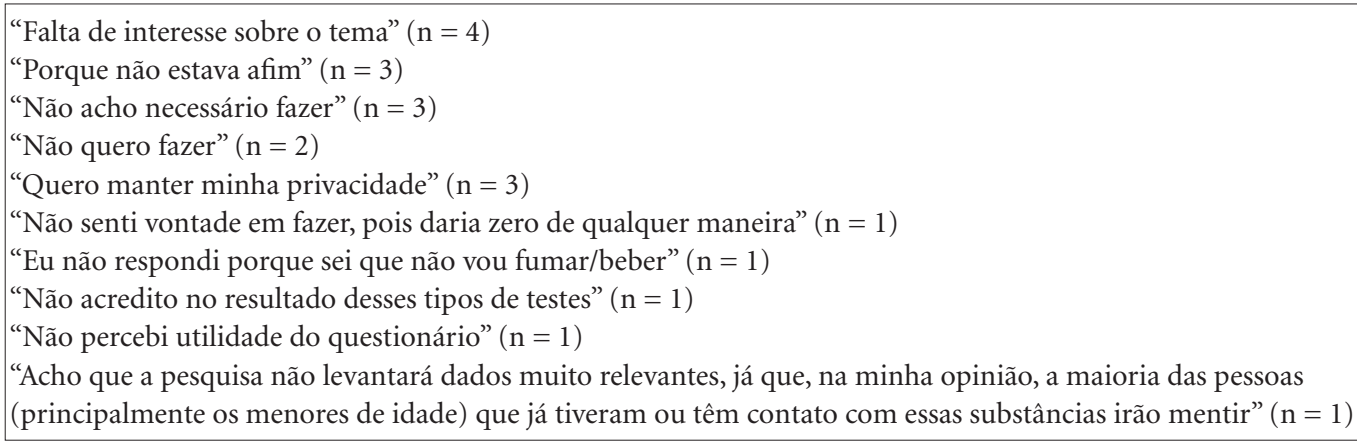

Tabela 1. Comentários e sugestões feitas pelos alunos $(\mathrm{N}=35)$.

\begin{tabular}{lrr}
\hline \multicolumn{1}{c}{ Tipo } & N & $\%$ \\
\hline Questões pouco específicas & 10 & 27,5 \\
Crítica sobre a mesma questão do CRAFFT/CESARE abordar álcool e outras SPA & 10 & 27,5 \\
Perguntar o que influenciou o uso de SPA & 2 & 6,0 \\
Perguntas "constrangedoras" & 1 & 3,0 \\
Questões pouco elaboradas & 1 & 3,0 \\
Generalização da questão B1 & 1 & 3,0 \\
Reformulação da questão B3 para que todos respondessem & 1 & 3,0 \\
Questão B6 muito subjetiva & 1 & 3,0 \\
Questões pouco claras & 1 & 3,0 \\
Melhor definição do que é considerado droga & 1 & 3,0 \\
Perguntas óbvias e que não consideram o aspecto social da pessoa & 1 & 3,0 \\
Perguntar sobre quantidade no caso de uso de álcool & 1 & 3,0 \\
Perguntar com período maior que 12 meses & 1 & 3,0 \\
Perguntar sobre frequência do uso de SPA & 1 & 3,0 \\
Perguntar Parte B apenas se a pessoa fez uso de SPA & 1 & 3,0 \\
Colocar as observações da Parte A embaixo & 1 & 3,0 \\
Total & 35 & $100 \%$ \\
\hline
\end{tabular}

Ao utilizar os demais pontos de corte (1, 3 e 4), foi observado respectivamente, especificidade de $92 \%$ e sensibilidade de $48,3 \%$ (Kappa 0,439); especificidade de 52,9\% e sensibilidade de $91,4 \%$ (Kappa 0,277); especificidade de $2,8 \%$ e sensibilidade de $99,1 \%(0,008)$.

Ao utilizar diferentes pontos de corte na Parte B, foi observado que com aquele igual a 3, 30,5\% dos estudantes apresentaram maior risco de ter qualquer problema relacionado ao uso, abuso e dependência de SPA; para o correspondente a 4 (ao menos uma resposta positiva na Parte A e quatro respostas afirmativas na Parte B), 20,2\% foram positivos. O ponto de corte 5 contou com $10,3 \%$ dos alunos e o correspondente a 6 apontou para 5,3\% de alunos com grande risco de dependência química.
A inspeção visual da curva ROC (Figura 2) revelou que o melhor ponto de corte foi maior ou igual a 2, tendo como produto a melhor relação entre sensibilidade e especificidade.

\section{Discussão}

O questionário CRAFFT/CESARE tem sido utilizado em diversas culturas e países como, por exemplo, EUA ${ }^{12,21}$, Canadá, França ${ }^{18}$, Suíça ${ }^{19}$, Alemanha $^{17}$, Singapura ${ }^{16}$, Noruega $^{14}$ e Colômbia ${ }^{15}$. O presente estudo corrobora a utilização do CRAFFT/CESARE como uma possibilidade de instrumento de triagem de uso de SPA por adolescentes em função de sua agilidade na aplicação, boa compreensibilidade e capacidade psicométrica. 
O questionário é breve, de simples pontuação, pode ser autoaplicável ou aplicado por profissional na atenção básica de saúde. Não é

Tabela 2. CRAFFT/CESARE positivo e CEBRID.

\begin{tabular}{lrrr}
\hline & \multicolumn{2}{c}{ CEBRID positivo } & \\
\cline { 2 - 3 } & Não & Sim & Total \\
& & & \\
CRAFFT/CESARE positivo & & & \\
Não & 313 & 15 & 328 \\
Soma & $95,4 \%$ & $4,6 \%$ & $100 \%$ \\
\%CRAFFT positivo & $73,3 \%$ & $12,9 \%$ & $60,4 \%$ \\
\%CEBRID positivo & $57,6 \%$ & $2,8 \%$ & $60,4 \%$ \\
\%Total & & & \\
Sim & 114 & 101 & 215 \\
Soma & $53,0 \%$ & $47,0 \%$ & $100 \%$ \\
\%CRAFFT positivo & $26,7 \%$ & $\mathbf{8 7 , 1} \%$ & $39,6 \%$ \\
\%CEBRID positivo & $21,0 \%$ & $18,6 \%$ & $39,6 \%$ \\
\%Total & & & \\
Total & 427 & 116 & 543 \\
Soma & $78,6 \%$ & $21,4 \%$ & $100 \%$ \\
\%CRAFFT positivo & $100 \%$ & $100 \%$ & $100 \%$ \\
\%CEBRID positivo & $78,6 \%$ & $21,4 \%$ & $100 \%$ \\
\%Total & & &
\end{tabular}

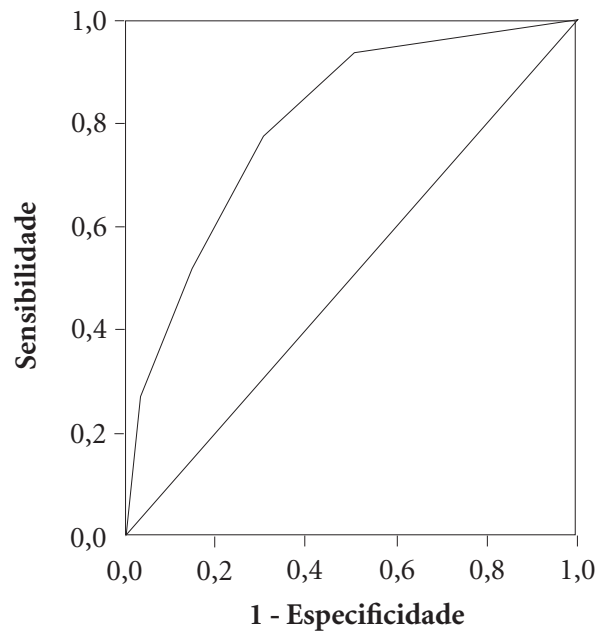

Área sob a curva $\mathrm{ROC}=0,802$

\begin{tabular}{ccc} 
Resultado & Sensibilidade & Especificidade \\
\hline 1,5 & 0.93 & 0.49 \\
$2,5^{*}$ & 0.77 & 0.69 \\
3,5 & 0.51 & 0.85 \\
4,5 & 0.27 & 0.96 \\
5,5 & 0.06 & 0.99
\end{tabular}

Figura 2. Curva ROC para o CRAFFT/CESARE. necessário qualquer tipo de treinamento para utilizá-la ${ }^{15}$. Diferentemente de outros instrumentos de triagem de uso de SPA por adolescentes, $\mathrm{o}$ CRAFFT/CESARE aborda questões referentes ao uso de substâncias lícitas e ilícitas ${ }^{12}$, além de incluir uma questão sobre trânsito e embriaguez ${ }^{12}$.

A autoaplicação do questionário possibilitou uma avaliação da compreensibilidade do instrumento, havendo uma taxa muito pequena $(2,2 \%)$ de estudantes que apontou dificuldades com o instrumento.

A maior parte dos comentários feitos refere-se a aspectos amplos sobre o tema, como, por exemplo, "perguntas constrangedoras" e "melhor definição do que é droga". Alguns estudantes fizeram sugestões tais como não englobar álcool e demais SPA nas mesmas questões (Parte B), propor que todos respondessem sobre usar sozinho e definir melhor a quantidade de álcool.

A crítica sobre englobamento de todas as SPA na mesma questão pode refletir uma percepção dos adolescentes de que o álcool não é uma substância potencialmente nociva à saúde, sendo até mesmo não classificado como droga por adolescentes em estudo recente ${ }^{34}$. Outro estudo nacional apontou que a percepção que o adolescente tem sobre as consequências nocivas do uso de álcool não acompanha a hierarquia dos prejuízos considerados mais graves ${ }^{35}$.

Cabe ressaltar que embora interessantes, as sugestões dos estudantes foram em número muito pequeno e em alguns casos fugindo ao escopo de um instrumento de triagem, portanto, consideramos não haver justificava para alterar a escala proposta pelo autor, já consagrada em diversos estudos $^{112,14-17,20}$. Uma possível alteração da versão original modificaria, provavelmente a sua capacidade psicométrica, sugerimos que outros estudos possam avaliar a relevância de testar a separação das perguntas.

Embora o uso de tabaco seja epidemiológica e clinicamente relevante entre adolescentes ${ }^{3,4}$, é importante destacar que neste uso não foi avaliado posto que o instrumento utilizado não possui questões específicas para esse fim. Apesar disso, vale salientar que o tabagismo deve ser valorizado nos atendimentos envolvendo essa população ${ }^{13}$.

Em relação à confiabilidade, os estudos sinalizaram que a consistência interna (alfa de Cronbach) do instrumento foi moderada a alta. A confiabilidade encontrada neste estudo foi considerada boa, embora levemente inferior à 
encontrada por Levy et al. ${ }^{7}$ (Kappa: 0.66-1.0), provavelmente em decorrência do instrumento comparativo utilizado nesta pesquisa.

No presente estudo, a porcentagem de alunos com CRAFFT/CESARE positivo foi de 36,2\%. Isto indica que mais de um terço dos participantes não só utilizaram álcool e/ou demais SPA no último ano, como também vivenciaram algum tipo de consequência em decorrência de tal uso, como dirigir ou andar de carro com alguém sob efeito do álcool e/ou esquecimento e/ou percepção familiar/amigos de uso exagerado e/ou uso para relaxar e/ou encrencas. Outros estudos de validação e adaptação cultural da escala CRAFFT que utilizaram o ponto de corte de duas ou mais respostas afirmativas, apontaram diversos índices de triagem positiva na população de adolescentes. Na Noruega o questionário foi positivo em $21,2 \%$ da amostra de adolescentes ${ }^{14}$, na Colômbia foi positivo em $49,6 \%$ dos adolescentes ${ }^{15}$, na Argentina foi positivo em $29 \%$ dos adolescentes $^{26}$, na Alemanha foi positivo em 63,6\% dos adolescentes $^{17}$, nos EUA 25\% ${ }^{12}, 14,8 \%, 29,5 \%$, $24,2 \%{ }^{11}$ e $33 \%{ }^{20}$ dos participantes pontuaram positivamente dependendo da população estudada (adolescentes internados, estudantes, ambiente rural).

Os dados apresentados no presente estudo (36,2\% dos alunos utilizaram SPA no último e vivenciaram consequências associadas a esse uso) são compatíveis com os dados nacionais para estudantes nesta faixa etária. Resultados do VI Levantamento Nacional entre estudantes apontam que o uso no ano de álcool foi referido por $42,4 \%$ dos adolescentes e 10,6\% utilizaram outra SPA, excetuando-se o álcool e o tabaco ${ }^{4}$. Em relação aos estudantes de escola pública o uso no ano de qualquer SPA, excluindo o álcool e tabaco, foi de $9,9 \%$ e o uso no ano de álcool foi de $41,1 \%$. Pesquisa realizada em estudantes brasileiros de 14 a 19 anos sinalizou que $51,2 \%$ dos participantes usaram álcool nos últimos 12 meses, 11,3\% preencheram critério para abuso ou dependência e 5,4\% beberam em padrão de binge ao menos uma vez na semana. Em relação às SPA ilícitas, 3\% relataram o uso no ano ${ }^{36}$. Outro estudo nacional observou que $35 \%$ dos adolescentes brasileiros consomem álcool ao menos uma vez por ano ${ }^{1}$.

Em estudo realizado em 2009, foi observado que com ponto de corte maior ou igual a 3 , a sensibilidade foi de $82 \%$ e a especificidade de $78 \%{ }^{20}$. Em Singapura foi utilizado o ponto de corte maior ou igual a 1 com sensibilidade de $78 \%$ e especificidade de $76 \%{ }^{16}$. Em geral, os pontos de corte estudados variam de 1 a 4 a depender da população avaliada ${ }^{20}$. Muito embora, a recomendação permaneça sendo o ponto de corte igual ou maior a 2. Na América do Sul, foi observada uma sensibilidade que variou de $95 \%$ a $59 \%$ e especificidade de $83 \%$ a $88 \%$ para o ponto de corte maior ou igual a 2 na Colômbia ${ }^{15}$ e Argentina ${ }^{26}$, respectivamente. Nesse sentido, estudo realizado na Noruega concluiu que uma pontuação igual a uma reposta positiva é suficiente para uma investigação mais aprofundada, já para fins de pesquisa o ponto de corte igual ou superior a 2 é preferível ${ }^{14}$.

Em nosso estudo confirmou-se que a pontuação igual ou maior a 2 é o ponto de corte com melhor razão entre sensibilidade e especificidade

Algumas limitações merecem ser mencionadas. Os estudantes participantes da pesquisa eram alunos em colégio técnico público em que existe uma seleção através de prova para os ingressantes, o que pode limitar a extrapolação dos dados para a população geral de adolescentes. $\mathrm{O}$ simples viés de o adolescente estar frequentando uma escola pode afetar a representatividade da população estudada, problema também enfrentado por estudos de adaptação da escala CRAFFT em outros países ${ }^{14}$. Entretanto, não há razão para acreditar que a validade e funcionalidade da escala seja alterada de acordo com o grupo estudado, já que o questionário foi especificamente desenhado para identificar os adolescentes de risco nos serviços de atenção primária e cumpriu seu papel adequadamente nesse contexto ${ }^{20}$.

A correlação entre as questões do CRAFFT/ CESARE e do instrumento utilizado no Levantamento do CEBRID, particularmente na Parte $B$, permitiu apenas uma aproximação, visto que poucas questões tiveram correspondência suficiente e uma delas não teve qualquer possibilidade de aproximação, fato que pode ter influenciado, negativamente, no grau de concordância entre as escalas.

A despeito das limitações apontadas, o presente estudo indica que a versão brasileira do questionário CRAFFT/CESARE foi bem compreendida pela população estudada, possui propriedades psicométricas adequadas e características que viabilizam sua utilização dentro do contexto sociocultural brasileiro. Sua rápida aplicação (em torno de 2 minutos) e a possibilidade de emprego em diferentes formatos (autoaplicação, entrevista, computadorizada $)^{14,15}$, tornam-na uma ferramenta útil na avaliação do uso de substâncias psicoativas em adolescentes. 


\section{Colaboradores}

BAAX Pereira contribuiu para a concepção e planejamento do artigo. Realizou a coleta de dados e criação de banco de dados. Participou ativamente na interpretação dos dados, na elaboração do artigo e na análise do conteúdo. PCF Schram contribuiu no processo de tradução e retrotradução da escala CRAFFT/CESARE e participou ativamente na análise crítica do conteúdo do artigo. RCS Azevedo contribuiu para a concepção e planejamento do artigo. Desempenhou importante papel na interface com as escolas, viabilizou a coleta de dados e a criação de banco de dados. Participou ativamente na interpretação dos dados, na elaboração do artigo e na análise do conteúdo.

\section{Referências}

1. Pinsky I, Sanches M, Zaleski M, Laranjeira R, Caetano R. Patterns of alcohol use among Brazilian adolescents. Rev Brasil Psiq 2010; 32(3):242-249.

2. Silva CC, Costa MCO, Carvalho RC, Amaral MTR, Cruz NLA, Silva MR. Iniciação e consumo de substâncias psicoativas entre adolescentes e adultos jovens de Centro de Atenção Psicossocial Antidrogas/CAPS-AD. Cien Saude Colet 2014; 19(Supl. 3):737-745.

3. National Institute on Drug Abuse (NIDA). Preventing Drug Use Among Children and Adolescents - A Research -Based Guide. Bethesda: NIH Publication; 2003.

4. Carlini EA, Noto AR, Sanchez ZM. VI Levantamento Nacional sobre o Consumo de Drogas Psicotrópicas entre Estudantes do Ensino Fundamental e Médio das Redes Pública e Privada de Ensino nas 27 Capitais Brasileiras: 2010. São Paulo: Centro Brasileiro de informações sobre Drogas psicotrópicas, UNIFESP; 2010.

5. Laranjeira R, Madruga CS, Pinsky I, Zaleski M, Caetano R, Mitsuhiro SS. II Levantamento Nacional sobre os Padrões de Consumo de álcool na População Brasileira. Brasília: Secretaria Nacional Antidrogas; 2012.

6. Brasil. Ministério da Saúde (MS). Secretaria de Atenção em Saúde. Departamento de Ações Programáticas Estratégicas. Diretrizes nacionais para a atenção integral à saúde de adolescentes e jovens na promoção, proteção e recuperação da saúde. Brasília: MS; 2010.

7. Levy S, Sherritt L, Gabrielli J, Shrier LA, Knight JR. Screening adolescents for substance use-related highrisk sexual behaviors. J Adolesc Health 2009; 45(5):473477.

8. Centers for Disease Control and Prevention (CDC). Youth Risk Behavior Surveillance - United States, 2009. Surveillance Summaries. MMWR 2010; 59:(SS-5).

9. Vieira DL, Ribeiro M, Romano M, Laranjeira RR. Álcool e adolescentes: estudo para implementar políticas municipais. Rev Saude Publica 2007; 41(3):396-403.

10. Tavares BF, Béria JU, Lima MS. Fatores associados ao uso de drogas entre adolescentes escolares. Rev Saude Publica 2004; 38(6):787-796.

11. Knight JR, Harris S, Sherritt L, Hook SV, Lawrence N, Brooks T, Carey P, Kossack R, Kulig J. Prevalence of positive abuse screen results among adolescents primary care patients. Arch Pediatr and Adolesc Med 2007; 161(11):1035-1041.

12. Knight JR, Sherritt L, Shrier LA, Harris SK, Chang G. Validity of the CRAFFT Substance abuse screening test among adolescent clinic patients. Arch Pediatr Adolesc Med 2002; 156(6):607-614.

13. Brasil. Ministério da Saúde (MS). Secretaria de Atenção à Saúde. Departamento de Ações Programáticas Estratégicas. A saúde de adolescentes e jovens: uma metodologia de auto-aprendizagem para equipes de atenção básica de saúde: módulo básico. Brasília: Editora do Ministério da Saúde; 2007.

14. Skogen JC, Bøe T, Knudsen AK, Hysing M. Psychometric properties and concurrent validity of the CRAFFT among Norwegian adolescents. Ung@hordaland, a population-based study. Addict Behav 2013; 38(10):2500 2505. 
15. Cote-Menendez M, Uribe-Isaza MM, Prieto-Suárez E. Validación para Colombia de la escala CRAFFT para tamización de consumo de sustancias psicoactivas en adolescentes. Rev. Salud Pública 2013; 15(2):220-232.

16. Subramaniam M, Cheok C, Verma S, Wong J, Chong SA. Validity of a brief screening instrument-CRAFFT in a multiethnic Asian population. Addict Behav 2010; 35(12):1102-1104.

17. Tossman, P, Kasten, L, Lang, P, Strüber, E. Bestimmung der konkorruentenValität des CRAFFT-d. $Z$ Kinder Jugendpsychiatr Psychother 2009; 37(5):451-459.

18. Karila L, Legleye S, Beck F, Corruble E, Falissard B, Reynaud M. Validation d'un questionnaire de repérage de l'usagenocif d'alcool et de cannabis dans la population générale: le CRAFFT-ADOSPA. Presse Med 2007; 36(4 Pt 1):582-590.

19. Bernard M, Bolognini M, Plancherel B, Chinet L, Laget J, Stephan P, Halfon O. French validity of two substance-use screening tests among adolescents: a comparison of the CRAFFT and DEP-ADO. Journal of Substance Use 2005; 10(6):385-395.

20. Dhalla S, Zumbo BD, Poole G. A review of the psychometric properties of the CRAFFT instrument: 19992010. Curr Drug Abuse Rev 2011; 4(1):57-64.

21. Cummins LH, Chan KK, Burns KM, Blume AW, Larimer M, Marlatt GA. Validity of the CRAFFT in American-Indian and Alaska-Native adolescents: screening for drug and alcohol risk. J Stud Alcohol 2003; 64(5):727-732.

22. Chang G, Orav EJ, Jones JA, Buynitsky T, Gonzalez S, Wilkins-Haug L. Self-Reported alcohol and Drug Use in Pregnant Young Women: A Pilot Study of Prevalence and Associated Factors. J Addict Med 2011; 5(3):221226.

23. Johnson KN, Raetz A, Harte M, McMahon LE, Grandsoult V, Garcia-Filion P, Notrica DM. Pediatric trauma patient alcohol screening: A 3 year review of screening at a Level I. J Pediatr Surg 2014; 49(2):330-332.

24. Pilowsky DJ, Wu L-T. Screening instruments for substance use and brief interventions targeting adolescents in primary care: A literature review. Addictive Behaviors 2013 (38):2146-2153.

25. Massachusetts Department of Public Health Bureau of Substance Abuse Services. Providers Guide: Adolescent Screening, Brief Intervention, and Referral to Treatment for Alcohol and Other Drug Use - Using the CRAFFT Screening Tool. Massachusetts: EOHHS; 2009.

26. Bertini MC, Busaniche J, Baquero F, Eymann A, Krauss M, Paz M, Catsicaris C. Transcultural adaptation and validation of the CRAFFT as a screening test for problematic alcohol and substance use, abuse and dependence in a group of Argentine adolescents. Arch Argent Pediatr 2015; 113(2):114-118.

27. De Micheli D. Uso de drogas por adolescentes: adaptação e validação de um instrumento de triagem (DUSI) e estudo das razões do uso inicial [tese]. São Paulo: Escola Paulista de Medicina; 2000.
28. Kelly TM, Donovan JE, b, Chung T, Bukstein OG, Cornelius JR. Brief screens for detecting alcohol use disorder among 18-20 year old young adults in emergency departments: Comparing AUDIT-C, CRAFFT, RAPS4QF, FAST, RUFT-Cut, and DSM-IV 2-Item Scale. Addictive Behaviors 2009; (34):668-674.

29. Knight JR, Sherritt L, Harris SK, Gates EC, Chang G. Validity of brief alcohol screening tests among adolescents: a comparison of the AUDIT, POSIT, CAGE, and CRAFFT. Alcohol Clin Exp Res 2003; (1):67-73.

30. Cook RL, Chung T, Kelly TM, Clark DB. Alcohol Screening in Young Persons Attending a Sexually Transmitted Disease Clinic Comparison of AUDIT, CRAFFT, and CAGE Instruments. J Gen Intern Med 2005; 20(1):1-6.

31. Pereira BAAX. Avaliação da versão brasileira da escala CRAFFT/CESARE de triagem de adolescentes para uso de álcool e outras drogas [dissertação]. Campinas (SP):Unicamp; 2014.

32. Schram PCF, Levy S. Triagem e Intervenção Breve em Abuso de Drogas. As Melhores Práticas para Especialistas. In: Sih T, organizador. IX Manual de Otorrinolaringologia Pediátrica da IAPO. São Paulo: Lis Gráfica e Editora; 2010. p. 19-27.

33. Carlini-Cotrim B, Carlini EA, Silva-Filho AR, Barbosa MTS. O uso de drogas psicotrópicas por estudantes de primeiro e segundo graus da rede estadual, em dez capitais brasileiras, 1987. In: Carlini-Cotrim B, Carlini EA, Silva-Filho AR, Barbosa MTS. Consumo de Drogas Psicotrópicas no Brasil em 1987. Centro de Documentação. Brasília, DF: Ministério da Saúde; 1989.

34. Rozin L, Zagonel IPS. Adolescentes que fazem uso nocivo/abusivo de álcool: percepção de risco e proteção para dependência. Rev. Eletr. Enf. 2013; 15(3):687-695.

35. Pechansky, F, Szobot, CM, Scivoletto, S. Uso de álcool entre adolescentes: conceitos, características epidemiológicas e fatores etiopatogênicos. Rev. Bras. Psiquiatr 2004; 26(1):14-17.

36. Madruga CS, Laranjeira R, Caetano R, Pinsky I, Zaleski $\mathrm{M}$, Ferri CP. Use of licit and illicit substances among adolescents in Brazil - a national survey. Addict Behav 2012; 37(10):1171-1175.

Artigo apresentado em 21/09/2014

Aprovado em 16/06/2015

Versão final apresentada em 18/06/2015 